Cinémas

Revue d'études cinématographiques

Journal of Film Studies

\title{
Convergences et divergences structurelles entre le processus de production et de réalisation d'un film et celui d'une exposition muséale
}

\section{Esther Pelletier}

Volume 6, numéro 1, automne 1995

URI : https://id.erudit.org/iderudit/1000964ar

DOI : https://doi.org/10.7202/1000964ar

Aller au sommaire du numéro

Éditeur(s)

Cinémas

ISSN

1181-6945 (imprimé)

1705-6500 (numérique)

Découvrir la revue

Citer cet article

Pelletier, E. (1995). Convergences et divergences structurelles entre le processus de production et de réalisation d'un film et celui d'une exposition muséale. Cinémas, 6(1), 151-168. https://doi.org/10.7202/1000964ar
Résumé de l'article

À l'ère pluridisciplinaire, avec les transferts et les échanges opérés d'un mode d'expression à un autre, la muséologie s'intéresse elle aussi au cinéma, au scénario et au système langagier narratif cinématographique. Cet article se veut un premier travail de débroussaillage et d'élaboration des premières pistes d'analyse des rapports convergents et divergents entre la production d'une exposition thématique muséale et celle d'un film. Sont ici comparés les étapes de développement des deux processus de production (film et exposition), leurs matériaux expressifs et leurs systèmes langagiers. 


\section{Convergences et divergences structurelles entre le processus de production et de réalisation d'un film et celui d'une exposition muséale}

\section{Esther Pelletier}

\section{RÉSUMÉ}

À l'ère pluridisciplinaire, avec les transferts et les échanges opérés d'un mode d'expression à un autre, la muséologie s'intéresse elle aussi au cinéma, au scénario et au système langagier narratif cinématographique. Cet article se veut un premier travail de débroussaillage et d'élaboration des premières pistes d'analyse des rapports convergents et divergents entre la production d'une exposition thématique muséale et celle d'un film. Sont ici comparés les étapes de développement des deux processus de production (film et exposition), leurs matériaux expressifs et leurs systèmes langagiers.

\section{ABSTRACT}

In this multi-disciplinary era, when transfers and exchanges between different modes of expression are common, museology has developed a new interest in the cinema, in the scenario, and in the linguistic system of cinematographic narrative. This article offers a preliminary exploration of the relationship between the production of a thematic museum exhibition and that of a film, and seeks to open avenues for further analysis. The elements compared include the developmental stages in the production processes for films and exhibitions, their expressive materials, and their linguistic systems. . 


\section{L'ère pluridisciplinaire}

Les études sur le cinéma accordent aujourd'hui une place importante au scénario comme objet d'analyse. Les récents colloques et publications ${ }^{1}$ sur le sujet en témoignent. L'écriture du scénario est devenue une étape clé, reconnue comme essentielle dans le développement d'un projet de film. Le cinéma étant un des arts et des moyens d'expression les plus populaires sur la planète, et vu l'éclatement des disciplines, les transferts et les échanges opérés d'un mode d'expression à un autre, la muséologie s'intéresse elle aussi au cinéma, donc au scénario et au système langagier narratif cinématographique.

Avec le cinéma, qui au départ fait appel à plusieurs matières de l'expression comme l'image, les bruits, la musique, les mentions écrites et les dialogues, avec le théâtre de recherche et la danse comme les ont pratiqués Robert Lepage avec Le Repère et Edouard Locke avec La La La Human Steps, le musée thématique est maintenant aussi devenu un lieu où l'usage de matériaux expressifs pluridisciplinaires contribue à l'organisation de ses expositions, véhicules de langage et de sens. En effet, l'exposition thématique, contrairement à l'exposition traditionnelle de tableaux ou de sculptures, par exemple, comporte une intention de récit et donc une organisation narrative qui s'appuie sur l'agencement plus ou moins logique et omnidirectionnel, comme on le verra plus loin, d'un faisceau d'éléments de l'expression langagière.

L'analyse qui va suivre et les rapprochements qui sont faits entre le cinéma et la muséologie sont tout a fait inusités. Il s'agit ici d'un travail de débroussaillage et d'élaboration des premières pistes qui, nous l'espérons, porteront fruit, permettant à d'autres chercheurs de s'interroger sur cette nouvelle question.

\section{Quand le commissaire d'expositions devient réalisateur}

Dans leur article "Du conservateur de musée à l'auteur d'expositions: l'invention d'une position singulière ", Nathalie Heinich et Michael Pollak, tous deux chercheurs au CNRS et membres du Groupe de sociologie politique et morale de l'institut d'Histoire du temps présent, se penchent, dans une étude commandée par le centre Georges Pompidou, sur le statut du 
conservateur ou commissaire d'expositions et sur l'organisation d'un certain type d'exposition comme celle de "Vienne, naissance d'un siècle» tenue à Beaubourg en 1986, et affirment :

Le cas du cinéma $[\ldots]$ paraît particulièrement adapté à une [...] comparaison: en tant, d'une part, que la notion d'auteur s'y est développée avec un relief particulier et que, d'autre part, les caractéristiques économiques de la production de films ont de nombreux points communs avec celles qui président à la production des expositions. On a affaire en effet, dans l'un et l'autre cas, à ce qu'on pourrait appeler une économie des produits culturels temporaires à grande diffusion, mais aussi à une économie de prototype, vouée à la fabrication d'un objet unique, susceptible certes de reproduction (cinéma) ou d'itinérance (exposition), mais pas de mise en série, puisque même si une certaine expérience peut être accumulée et réutilisée d'un produit à l'autre, il n'y a pas de véritable standardisation, ni des budgets, ni du personnel, ni des matériaux. Enfin, dans l'un et l'autre cas, on trouve la conjonction d'un travail en équipe dirigé par un maître d'œuvre, l'identité de ce dernier pouvant $[\ldots]$ subir d'importantes variations: producteur, scénariste, réalisateur, conservateur maison ou commissaire spécialisé, concepteur ou architecte [...] (p. 39).

\section{Ils ajoutent :}

[...] par-delà la question de la personne, l'accès à une position d'auteur fait appel à d'autres conditions. Nous en distinguerons trois ici, dont on pourrait montrer qu'elles jalonnèrent l'évolution de la critique de cinéma. La première est la mise en évidence d'une thématique, autrement dit d'une unité de préoccupations personnelles investie au niveau du contenu de l'œuvre, voire sur plusieurs œuvres. La seconde est la mise en évidence d'une stylistique, autrement dit d'une unité dans le parti pris de mise en forme, repérable là encore à l'intérieur d'une même œuvre ou - mieux encore d'une œuvre à l'autre. La troisième caractéristique, enfin, de cette accession au statut d'auteur, réside dans le registre de réception de l'auvre par le public (du public spécialisé des critiques au grand public), perçue 
dans son "opacité", comme une œuvre à part entière, faisant l'objet d'un jugement spécifique [...] (p. 4445).

Comme on le voit, le développement d'un certain type d'exposition où foisonnent plusieurs modes d'expression peut se comparer au développement d'un projet de film mettant comparativement en jeu les mêmes conditions de production, le même type d'intervenants et, hypothétiquement, une certaine similitude de l'organisation langagière en termes de thématique, de stylistique et de réception de l'œuvre. C'est ce que nous tenterons maintenant de comparer en analysant le processus habituel de développement d'un film et celui de l'exposition permanente Mémoires du Musée de la civilisation de Québec, créée en 1988 à l'ouverture de ce musée. Cette exposition a ceci de particulier qu'elle avait et a toujours pour fonction de situer ce musée dans le vaste champ de la civilisation, de tenter d'expliquer ce qu'est l'identité culturelle, ce qu'est la société "distincte", de situer la civilisation québécoise en tenant compte de ses composantes multiethniques au moyen d'une approche qui ne tient pas uniquement compte de la civilisation canadienne-française catholique, mais aussi des communautés anglophone, écossaise, irlandaise et des immigrants italiens, juifs, vietnamiens, etc.

Dans le cadre de cet article, nous aborderons les trois points suivants: 1) les étapes de développement d'un projet de film et d'un projet d'exposition thématique; 2) les matériaux expressifs; 3) l'organisation langagière.

\section{Les étapes de développement d'un projet de film et d'un projet d'exposition thématique}

Ici, nous comparerons les étapes d'un projet de film destiné aux salles de cinéma et celles de l'exposition permanente Mémoires du Musée de la civilisation de Québec. Cette exposition nous est apparue représentative de l'ensemble des expositions d'un musée thématique et comparable au développement d'un projet de film, qui exprime aussi une ou plusieurs idées thématiques. 


\section{TABLEAU 1}

Processus DE PRODUCTION D'UN FILM ET D'UNE EXPOSITION FILM EXPOSITION

\section{ORIGINES DES PROJETS}

\section{INDIVIDU(S)}

- Auteur (scénariste, réalisateur)

- Producteur

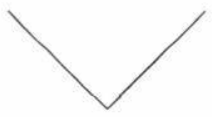

Vision d'un individu

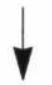

Doit répondre aux critères de rentabilité culturelle et économique des sociétés d'État qui financent entre $85-90 \%$ du projet

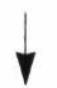

Financement d'État géré par les sociétés d'État et le producteur à qui on consent la gestion du financement

\section{INSTITUTION(S)}

- Le musée d'accueil

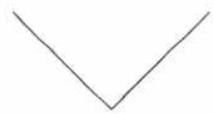

Objectifs des orientations du musée

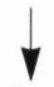

Vocation culturelle

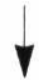

Financement d'État géré par l'institution qui motive le projet (le musée)

\section{PREMIËRE ÉTAPE}

- Image, flash, scène, person- $\mid$ - IDÉES nage(s), idée(s)

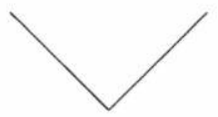

$$
\text { | }
$$

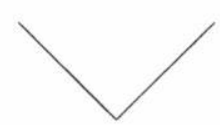

Convergences et divergences structurelles entre le processus de production... 


\section{SUJET et / ou \\ SYNOPSIS}

- Recherche de financement par le producteur

- Produit par l'auteur
Document d'orientations et stratégie de communications

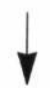

- Définir la surface d'exposition et le budget

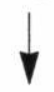

- Produit par les intervenants du musée et des consultants externes (18 mois)

\section{DEUXIÈME ÉTAPE}

- Écriture du scénario (Financement trouvé) (3-5 versions ou plus, 18-24 mois)
- Démarche conceptuelle

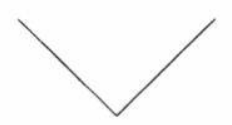

- Établissement du concept

Choix des thèmes et des sous-thèmes

Choix de l'angle de traitement (ambiances), esquisse du fil conducteur, choix des collections associées à chaque bloc thématique (3 mois)

\section{TROISIÈME ETAPE}

- Préproduction

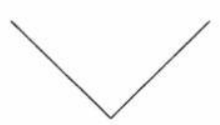

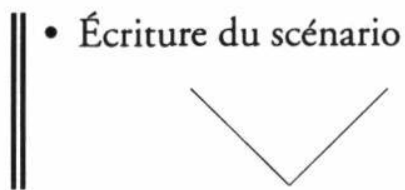


Établissement des besoins humains, matériels et techniques à partir de :

- traitement cinématographique

- découpage technique

- dépouillement

- plan de travail
- Établir ce que l'exposition a à raconter en s'appuyant sur la recherche

- Énoncer clairement le parcours à pied linéaire que le visiteur suivra (3 mois)

\section{QUATRIÈME ÉTAPE}

- La réalisation

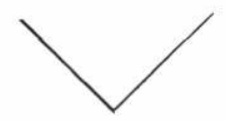

Tournage

Montage

Sonorisation

Copie zéro ( 8 mois)
- La réalisation

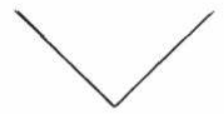

Mise en espace des objets et des supports techniques et technologiques (6 mois)

Dans le cas du cinéma, le film sert à exprimer la vision d'un auteur (scénariste et/ou réalisateur) et/ou d'un producteur. Il doit également répondre aux objectifs économiques et culturels des sociétés d'État qui financent le projet, du moins dans les pays où sont établies de telles structures, ce qui est le cas au Québec et au Canada. Donc, l'origine de l'idée dans le cas du cinéma vient d'un auteur ou d'un producteur.

En ce qui a trait à l'exposition muséale, celle-ci doit répondre aux objectifs des orientations que s'est fixés le musée qui la motive. Dans le cas de l'exposition Mémoires qui, à l'origine, se voulait l'exposition permanente du Musée de la civilisation de Québec, celle-ci devait pouvoir non seulement permettre de situer ce musée dans le vaste champ de la civilisation, mais elle devait aussi servir l'image d'une institution à vocation culturelle. Ici, comme on le voit, l'origine du projet est motivée par 
l'institution d'accueil qui le développe et le finance à même le budget annuel que lui attribue l'État.

\section{Les étapes de développement}

\section{- PREMIÈRE ÉTAPE}

La première étape que doit franchir un projet de film est celle du développement d'une image, d'un flash, d'une scène, d'un personnage ou d'une idée qui vient à l'esprit de l'auteur (ou du producteur) et qui aura, pour forme première narrative, celle d'un sujet et d'un synopsis embryonnaires. Avec ces premiers textes, s'établit la première continuité narrative organisée dans ses grandes lignes. Une description des personnages accompagne habituellement ces premiers documents. Ceci permet d'avoir une meilleure idée de l'ensemble de l'histoire et permet au producteur de chercher du financement pour écrire le scénario de l'histoire.

Quant à la première étape de développement d'un projet d'exposition muséale, elle consiste à réunir les responsables de l'exposition du musée et quelques consultants externes, de manière à établir un premier document d'orientations d'où découlera une stratégie de communication débouchant sur l'utilisation de l'espace. Une fois connue l'utilisation de la surface d'exposition, le budget pourra être établi.

— DEUXIÈME ÉTAPE

Une fois le financement trouvé, la deuxième étape de développement d'un film consiste à écrire le scénario de l'histoire en une, deux, trois, quatre versions ou parfois davantage. L'écriture du scénario d'un long métrage peut facilement prendre de 18 à 24 mois, et tout au long de son développement, le traitement cinématographique de l'histoire commence à poindre dans l'écriture. C'est également vers la fin de cette étape que le producteur, scénario en main, procède à la recherche du financement de la production du film.

La deuxième étape de production d'une exposition muséale, quant à elle, differe de celle du film. Alors que pour ce dernier, le scénario en est à l'étape de l'écriture et que le traitement cinématographique n'est qu'embryonnaire, ici, on en est à définir la démarche conceptuelle, le choix de l'angle de traitement (par 
exemple le choix des ambiances), l'établissement définitif du concept, des thèmes et des sous-thèmes, de même qu'une esquisse du fil conducteur. Le choix des collections associées à chaque bloc thématique fait également partie de cette étape où l'on ne parle pas encore de scénario.

— TROISIÈME ÉTAPE

Une fois le devis de production établi à partir de la dernière version du scénario de film et le financement du film trouvé, le producteur et son équipe procèdent à l'établissement des besoins humains (chez les comédiens et au sein de tous les secteurs de métiers requis), matériels (lieux, objets) et techniques (appareillages divers). Il réserve alors toutes les ressources nécessaires identifiées en fonction des besoins établis à partir du découpage technique, du traitement, du dépouillement et du plan de travail. Cette étape est vraiment celle que l'on appelle, dans le métier, l'étape de préproduction.

L'étape trois de l'exposition correspond à celle de l'écriture $d u$ scénario. C'est ainsi qu'on la nomme. Pour le chargé de projet ou commissaire d'expositions, il s'agit de définir ce que l'exposition a à raconter, en s'appuyant sur la recherche qui a été faite sur le sujet. Il s'agit également d'énoncer le plus précisément possible le parcours au pied linéaire que le visiteur va faire en suivant l'exposition.

- QUATRIÈME ÉTAPE

C'est ici que convergent les étapes de développement du processus de production d'un film et celles d'une exposition. L'étape quatre, dans les deux cas, aboutit à la réalisation concrète du produit après trois grandes étapes de préparation. Ici, on réalise le projet. Dans le cas du cinéma, il faut procéder au tournage, au montage et à la sonorisation avant d'obtenir la copie zéro du film, alors que pour l'exposition, il s'agit de mettre en place tous les objets et supports techniques ainsi que technologiques qui constitueront le parcours de l'exposition et son système langagier.

Comme on le voit, ces deux organisations, le film et l'exposition, se ressemblent, mais different également. 


\section{Les matériaux expressifs}

Au cinéma, lorsque le scénariste commence à échafauder le film, il dispose d'une série de moyens langagiers que lui fournit l'expression cinématographique: dialogues, bruits, musique, mentions écrites et images que l'on regroupe communément sous deux grandes catégories ou matières, celle de l'image et celle du son. Ces matières expressives trouvent à leur tour leur assise sur des matériaux expressifs qui, en quelque sorte, sont les paramètres de l'expression cinématographique à partir desquels l'histoire et le style cinématographiques se détermineront et d'où se joueront le sens et la communication avec le spectateur.

Le cinéma fait usage de plusieurs procédés techniques et éléments diversifiés pour parvenir à signifier. Tous ces matériaux expressifs, ces procédés et éléments, relèvent principalement de deux ordres, du filmographique et du profilmique. Par filmographique, nous entendons, à la suite d'Étienne Souriau ${ }^{2}$ et de Christian $\mathrm{Metz}^{3}$, tout ce qui se rapporte à la manipulation de l'appareillage cinématographique (au tournage, au montage, en laboratoire) et dont on peut voir les effets à l'écran lors de la projection; tandis que par profilmique, nous entendons tout ce qui apparaît devant la caméra au tournage.

Ainsi, à titre d'exemple, en ce qui concerne l'image, nous avons proposé une classification (voir tableau 2) des paramètres de l'expression cinématographique ${ }^{4}$.

À l'étape de l'écriture du scénario, certains de ces aspects peuvent être pris en considération par le scénariste pour le développement des scènes. Par exemple, au lieu de dire: "Marie descend l'escalier", il écrira: "Les jambes de Marie apparaissent dans l'escalier ", etc. On voit donc ici, par le choix d'une échelle de plans, l'inscription du découpage technique dans l'écriture, ce qui n'est pas une règle en soi, mais une possibilité d'écriture. Il revient donc au scénariste, à l'étape de l'écriture du scénario, puis au réalisateur et même au directeur de la photographie et au monteur, à l'étape de la production du film, d'utiliser ces matériaux expressifs. Ils les font permuter d'un scénario à l'autre et d'un film à l'autre, créant et renouvelant sans cesse leur expression et leur sens. Chaque scénario et chaque film deviennent ainsi des produits singuliers. 
D'autre part, si les matériaux expressifs sont identifiables, comme nous venons de le démontrer, leur utilisation en cours de traitement cinématographique ne peut cependant être réglée selon des principes entendus. En arriver à une telle procédure contribuerait à limiter leurs possibilités expressives. Tout au plus, nous avancerons que leur utilisation crée des effets de sens et que l'auteur a donc à sa disposition plusieurs matériaux expressifs qu'il peut utiliser. Le résultat dépendra de ce qu'il en fera. Et enfin, son scénario et/ou son film seront uniques.

\section{TABleau 2}

\section{LE FILM : MATÉRIAUX EXPRESSIFS}

\section{A) Paramètres filmographiques}

\section{Image}

1. Le support
a) la pellicule
b) les photogrammes

2. La disposition spatiale
a) l'échelle des plans
b) les angles de prises de vue
c) les mouvements de caméra
d) le cadrage
e) la profondeur de champ

3. Le montage
a) alterné
b) parallèle
c) répétitif
d) l'insert
e) le flash-arrière
f) le flash-avant
g) le champ et le contrechamp

4. Les effets de montage
a) la coupe franche
b) le jump-cut 
5. Les effets spéciaux
a) le fondu
b) la surimpression
c) le volet
d) l'iris
e) le rideau
f) le cache et contrecache
g) autres effets spéciaux

6. La disposition temporelle
a) au niveau chronologique
- temps réel
- temps bouleversé
- temps aboli
b) au niveau chronométrique
- temps abrégé
- temps respecté
- temps allongé

B) Paramètres profilmiques

1. Les personnages

2. Les objets
a) décors
b) costumes
c) maquillage
d) autres objets

3. L'éclairage

C) Paramètres filmographiques et profilmiques

1. La couleur
a) filmographique
- type de pellicule couleur
- étalonnage
- exposition
b) profilmique
- la couleur des objets du film 
Si l'on se tourne maintenant du côté de l'exposition muséale, il faut d'abord dire que, selon les spécialistes ${ }^{5}$, la muséographie s'est au fil du temps développée au niveau des techniques de mise en espace; et si le cinéma est un médium en soi qui, comme on vient de le voir, a recours à plusieurs systèmes langagiers pour signifier (musique, bruits, images, mentions écrites, etc.), il en est de même de l'exposition muséale, qui utilise de nombreux systèmes langagiers pour se développer dans le temps et l'espace. Un premier inventaire nous a permis d'établir que les plus fréquemment employés sont les suivants:

\section{TABLEAU 3}

\section{EXPOSITION MUSÉALE : SYSTÈMES LANGAGIERS}

1) les objets et leur organisation dans l'espace;

2) la salle d'exposition ou sa volumétrie comme matériau expressif (éclairage comme modulateur spatial);

3) le son;

4) la vidéo (le documentaire qui fait intervenir des individus dans un univers d'objets);

5) l'image virtuelle;

6) le film;

7) la photographie ;

8) la scénographie de composition qui met en contexte des thèmes englobants;

9) les jeux interactifs (support informatique);

10) la musique.

Ces nombreux systèmes langagiers s'interpellent les uns les autres et, comme au cinéma, créent du sens en simultanéité de coprésence.

\section{L'organisation langagière}

Dans cette partie de notre analyse, nous aborderons les aspects de la thématique et de la stylistique que font ressortir Heinnick et Pollack lorsqu'ils comparent le cinéma et l'exposition muséale.

Convergences et divergences structurelles entre le processus de production... 163 


\section{- LA THÉMATIQUE}

Le scénario et le film sont fondamentalement l'illustration et la démonstration de thèmes inscrits dans un schéma narratif de développement. Celui-ci s'appuie sur des personnages et des situations qui s'enchaînent, permettant aux thèmes de s'actualiser au fur et à mesure que l'histoire se développe jusqu'à sa conclusion.

Par exemple, le film Les Plouffe (1981) de Gilles Carle, adapté du roman de Roger Lemelin (1948) et scénarisé par les deux auteurs, repose fondamentalement sur le thème de la famille canadienne française des années 30 et 40 , mise en relation avec la société de l'époque et ses institutions, de même que ses valeurs. C'est ainsi que l'Église, la religion, la morale et le pouvoir deviennent des sous-thèmes qui se greffent au thème central de la famille. Enfin, thèmes et sous-thèmes sont pris en charge par une galerie de personnages principaux et secondaires et un ensemble de situations qui les illustrent jusqu'à leur actualisation, considérée complète au moment du dénouement de l'his-

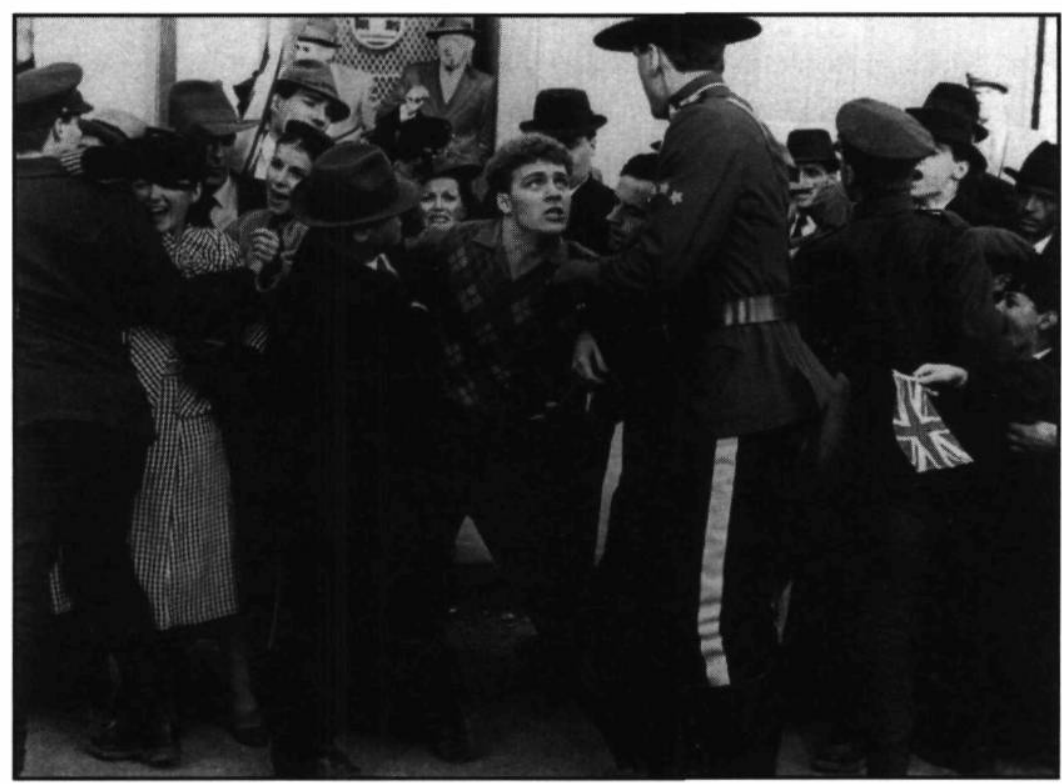

Les Plouffe de Gilles Carle (1981)

Collection Cinémathèque québécoise 
toire. Nous avons choisi ce film à titre d'exemple parce qu'il rejoint directement la thématique de l'exposition Mémoires.

En effet, celle-ci repose essentiellement sur le grand thème de l'identité culturelle québécoise exprimée à travers le traitement de la mémoire et ses sous-thèmes sont la nature, la culture, la famille, les institutions comme l'Église, l'école et l'État, le sport, la politique, les fêtes, les arts et les enjeux modernes de la société québécoise et de la culture.

Comme on le voit, non seulement dans leur démarche de développement (thème, image, idée, flash, concept), mais intrinsèquement, le film et l'exposition muséale sont le véhicule représentatif de thèmes et de sous-thèmes qui motivent leur traitement et leur développement. C'est ce que nous verrons à l'instant.

\section{- LA STYLISTIQUE}

Au cinéma, l'histoire prise en charge par les personnages qui génèrent l'action se développe sur un mode narratif constitué de segments qui s'enchaînent selon un certain ordre logique. Par exemple, dans le film Nikita (1990) de Luc Besson, qui nous apparaît être un film exemplaire permettant de saisir avec précision l'enchaînement logique des événements, il y a 11 grands segments d'action composés de près d'une centaine de segments médians qui illustrent le thème de l'épreuve à surmonter et le sous-thème de l'amour. Pour l'exposition Mémoires, il y a six zones distinctes qui prennent en charge le thème de l'identité culturelle et les sous-thèmes énumérés précédemment (voir tableau 4). Dans l'un et l'autre cas, film et exposition muséale, la stylistique de l'organisation langagière se manifeste à travers des niveaux d'organisation du sens. Aussi pour le scénario et le film, il y a trois niveaux d'organisation (grands, moyens et petits segments) alors que pour l'exposition muséale, les muséologues parlent de zones qui se divisent en scènes, et ces scènes en tableaux (voir tableaux 5 et 6 ).

Il y a donc dans le cas du film et de l'exposition, une organisation stylistique comparable. 
TABLEAU 4

\section{LA STYLISTIQUE}

Le film Nikita (1990)

de Luc Besson

segments narratifs

(Patterns)
L'exposition Mémoires

zones
1. L'attaque de la pharmacie
1. Le bon vieux temps par les junkies

2. L'interrogatoire de Nikita

2. Un pays à bâtir

3. Le procès

3. Des jours difficiles

4. La proposition

4. Les pouvoirs

5. L'entraînement

5. Ensemble

6. L'épreuve

6. Et maintenant

7. La vie dehors

8. La première mission

9. La seconde mission

10. La troisième mission

11. Les aveux et la disparition de Nikita

TABLEAU 5

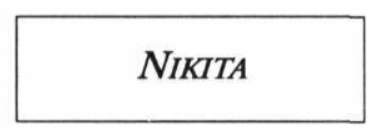

Segments narratifs

Pattern (grand segment)

1. L'attaque de la pharmacie par les junkies

Sets (segments médians)

1.1. Se rendre à la pharmacie

1.2. Chercher la clé

1.3. Défoncer la porte

1.4. Avertir les policiers

1.5. Charger les fusils

1.6. Etre découverts par le pharmacien

1.7. Arrivée des policiers 


\subsection{Attaque}

Isolats (petits segments)

1.8.1. Un junkie ouvre le feu et tue le pharmacien

1.8.2. Le pharmacien s'écroule

1.8.3. Les policiers mettent des masques de précision pour viser leurs cibles

1.8.4. Échanges de coups de feu

1.8.5. Le grand blond s'écroule

1.8.6. Rico s'écroule

1.8.7. Antoine s'écroule

Etc.

1.9. Constat des morts et des blessés

1.10. Découverte de Nikita vivante

1.11. Nikita tue le policier

TABleaU 6

MÉMOIRES

Zone 4

Les pouvoirs

\section{Scènes}

\subsection{L'Église}

4.2. Les pratiques religieuses

Tableaux

4.2.1. Tableau de piété

4.2.2. Vêtements liturgiques

4.2.3. Intérieur d'une église

4.2.4. Les communautés religieuses

4.3. L'école d'antan

4.4. L'école contemporaine

4.5. L'école (aire d'animation)

4.6. La citoyenneté

Il faut cependant noter qu'au cinéma, le spectateur est en présence d'un récit qui lui est imposé et dont il n'a pas le contrôle de l'organisation. Tandis qu'avec l'exposition muséale, le visiteur 
est en présence d'un parcours qu'il peut modifier à sa guise et dont il contrôle l'organisation jusqu'à un certain point. Ainsi, la lecture du récit au cinéma est-elle davantage unidirectionnelle alors que celle que fait le visiteur au moment de l'exposition est davantage omnidirectionnelle. On voit donc ici poindre de nouvelles ouvertures dans le champ des théories et de l'analyse du récit qui, de toute évidence, se complexifient avec la multiplication des lectures qu'engendrent les parcours d'expositions muséales, sans compter la multidisciplinarité des langages utilisés.

Université Laval

\section{NOTES}

1 Colloque de Bruxelles (1988), colloque de l'Association québécoise des études cinématographiques (AQEC) (1992); Esther Pelletier, Écrire pour le cinéma. Le Scénario et l'industrie du cinéma québécois (Québec: Nuit Blanche, 1992), "Le scénario", Cinémas, vol. 2, n l (1991), "Le scénario de film", Études littéraires, vol. 26, n 2 (1993).

2 Étienne Souriau, L'Univers filmique (Paris: Flammarion, 1953).

3 Christian Metz, Essais sur la signification au cinéma. Tome II (Paris: Klincksieck, 1972).

4 Esther Pelletier, Écrire pour le cinéma. Le Scénario et l'industrie du cinéma québécois (Québec: Nuit blanche, 1992).

5 Notamment le muséologue Philippe Dubé, professeur agrégé au Département d'histoire de l'Université Laval, avec qui nous avons pu discuter de ces questions.

OUVRAGE CITÉ

Heinich, Nathalie et Pollak, Michael. "Du conservateur de musée à l'auteur d'exposition : l'invention d'une position singulière ". Sociologie du travaih $\mathrm{n}^{\circ} 1,1990$. 